\title{
LDH Isoenzyme 5 to LDH Ratio Measurement
}

National Cancer Institute

\section{Source}

National Cancer Institute. LDH Isoenzyme 5 to LDH Ratio Measurement. NCI Thesaurus. Code C79455.

The determination of the ratio of LDH isoenzyme 5 compared to total LDH present in a sample. The measurement may be expressed as a ratio or percentage. 